EPJ Web of Conferences 59, 13002 (2013)

DOI: $10.1051 /$ epjconf/20135913002

(C) Owned by the authors, published by EDP Sciences, 2013

\title{
HIDEX: A new high resolution x-ray spectrometer for detailed line profile measurements
}

\author{
E. Galtier ${ }^{1,2, a}$, O. Renner ${ }^{3}$, E. Krouský ${ }^{3}$ and F. Rosmejej ${ }^{2,4}$ \\ ${ }^{1}$ SLAC National Accelerator Laboratory, LCLS, Menlo Park, California 94025, USA \\ 2 École Polytechnique, LULI, PAPD team, 91128 Palaiseau, France \\ 3 Institute of Physics of the ASCR, 182 21, Prague 8, Czech Republic \\ ${ }^{4}$ Sorbonne Université, LULI, UMR 7605, case 128, 75252 Paris Cedex 05, France
}

\begin{abstract}
We present preliminary obtained with a new spectrometer based on the Johann configuration of cylindrically bent crystals, the HIDEX. The aim of this instrument is to provide detailed line shape and shift measurements of transitions originating from high intensity laser/matter interaction, especially when matter is in extreme conditions of temperature and density. The HIDEX provides two new features. First, its alignment procedure has been improved being now based on an accurate motorized rotation stage that provides a robust and fast way to position the main components in the desired geometrical configuration. Second, there is the option to mount a Charge Coupled Device (CCD) as detector, allowing the instrument to be operated in high repetition rate laser facilities where opening the chamber migh be a critical issue. Here, we report about the test of the prototype at PALS kilo-joule laser facility, Prague, that demonstrated the new alignment procedure concept. First results are discussed.
\end{abstract}

\section{INTRODUCTION}

In the past few years, the newly developed $4^{\text {th }}$ generation light sources invoked great excitement in the scientific community due to their exceptional characteristics: femtosecond pulse length, high photon energy, low divergence, high beam energy, all of these parameters contributing to the unprecedented high peak brightness, about 10 orders of magnitude higher than for the previous generation. In plasma physics, this new tool can be used to create well defined warm dense matter (WDM) samples, state of matter of fundamental role in inertial confinement fusion (ICF) [1], astrophysics, planetary science [2], high intensity laser produced plasmas [3] and non equilibrium properties of matter [4]. Diagnosis using X-ray emission spectroscopy of such matter [5] reveals a complex and rich behaviour but fine effect whose origin stems from high electron density still remains unclear. This is mainly due to the lack of high resolution spectroscopy $\left(\frac{\lambda}{\Delta \lambda} \gtrsim 4000\right)$ observing WDM emission in the stringent experimental conditions required in X-ray laser facilities (low number of shifts, high repetition rate, long-run evacuated experimental chambers, ... ). In this paper, we present a prototype of a HIgh DispErsion X-ray spectrometer, the HIDEX, that gathers all of the requirement for such operation providing high spectral and spatial resolution that can resolve line profile or line shifts in WDM. First, we will present the prototype of the instrument and the experimental setup in which it has been preliminary tested. Second, we will give a brief description of the theory behind this spectrometer giving more explicit description of the reconstruction phase of a spectrum. Finally, early results are discussed before conceiving the conclusion.

\footnotetext{
ae-mail: egaltier@slac.stanford.edu
}

This is an Open Access article distributed under the terms of the Creative Commons Attribution License 2.0, which permits unrestricted use, distribution, and reproduction in any medium, provided the original work is properly cited. 


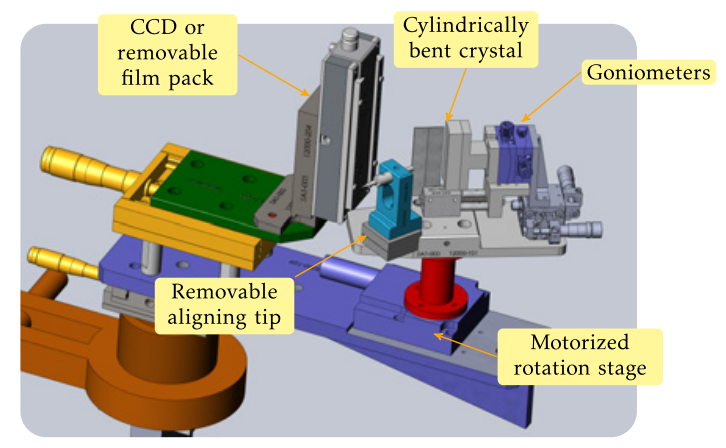

Figure 1. View of HIDEX spectrometer without protective covers. The three main components (the crystal holder, the detector holder and the alignment tips) are shown.

\section{THE INSTRUMENT AND THE EXPERIMENTAL SETUP}

The HIDEX is based on the Vertical Johann Spectrometer, first designed and successfully used over several years by O. Renner and coworkers [6]. The HIDEX uses a cylindrically bent crystal in a verticaldispersion Johann configuration with both the source and the detector on the Rowland circle in order to maximize the collection efficiency as well as the spectral and spatial resolutions. Due to its vertical orientation, the crystal diffracts radiations such that the spectral axis is vertical, while emission from other slices of an extended plasma source are resolved in the horizontal direction (the spatial axis). These combined effects produce bow like structures on the detector whose complex shape needs to be reconstructed non-linearly (see Sect. 3). Figure 1 shows a 3D view of the mechanical assembly of the HIDEX. Three main components can be clearly distinguished: the crystal holder, the detector holder and the alignment tips. First, the crystal holder uses three translations (XYZ) and two goniometers in order to precisely control the position and the tilt of the crystal. This is a key point since the crystal holder is mounted on a motorized rotation stage whose rotation axis needs to be coincident with the crystal vertical axis at its center. The use of such motorized stage allows to rapidly align the spectrometer while preserving a high accuracy required to achieve high spatial and spectral resolution. Second, the detector, whose center is placed on the Rowland circle, can be either an X-ray film, an Imaging Plate or a CCD. Both film and IPs (with an active area of $6 \times 1 \mathrm{~cm}^{2}$ ) are in a box that can be removed easily using precisely machined magnets. The CCD allows the use of the HIDEX in a high repetition rate environment that requires to keep the experimental chamber closed for a long time. Finally, a set of two removable tips are used to align the crystal on the rotation axis and to align the spectrometer with respect to the target. The intrinsic alignment of the spectrometer can be done offline using a dedicated breadboard and viewing system. All components are aligned within $10 \mu \mathrm{m}$ accuracy while the tilts of the crystal are set within $0.05^{\circ}$. These uncertainties are controlled using cameras that look at the alignment tip. When inside the chamber, it is then assumed that the spectrometer retains his internal geometry. In the Johann geometry, the radius of curvature of the crystal constrains the position of the spectrometer with respect to the source. Also, bent crystal of good quality and reasonable price add even more constrains. Therefore, an optimal distance, not too close to the target (to avoid debris on the crystal surface) but not too far (to cover a wide spectral range), has to be chosen (see Sect. 4).

\section{THEORY: A SURVEY}

The theory beyond the vertical Johann configuration has been extensively investigated in previous work [6]. Based on this study, we developed a software that reconstructs an image recorded on any detector. The robustness in the reconstruction process relies on the symmetry of the bow-like spectrum 


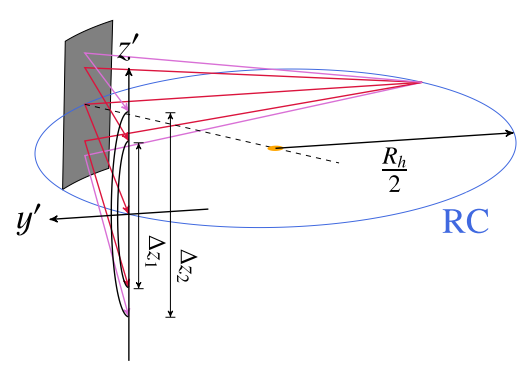

Figure 2. View of vertical Johann configuration geometry. $\mathrm{R}_{h}$ is the crystal bending radius equal to a diameter of the Rowland circle (RC). $z^{\prime}$ and $y^{\prime}$ are the spectral and spatial axis respectively. $\Delta z_{1}$ and $\Delta z_{2}$ are the spacing between the foot of the bows corresponding to spatially extended quasi-monochromatic emission of two spectral lines.

due to the vertical geometry of the crystal whose median horizontal plane coincides with the Rowland circle (see Figure 2).

Three equations describe the algorithm, which requires detection of at least two emission lines:

1. Eq. (1) gives the central wavelength using spacing $\Delta z_{1}$ and $\Delta z_{2}$ between the set of two symmetrical profiles which can be precisely measured within the software.

$$
\lambda_{0}=\sqrt{\left(\Delta z_{1}^{2} / \Delta z_{2}^{2}-1\right) /\left(\Delta z_{1}^{2} / \Delta z_{2}^{2} \lambda_{2}^{2}-1 / \lambda_{1}^{2}\right)}
$$

2. The corresponding Bragg angle in the horizontal plane is given by $\theta_{0}=\arcsin \left(\frac{\lambda_{0}}{2 d}\right)$ where $d$ is the crystal interplanar spacing.

3. Mapping of the spectra is done using eq. (2). Each increment in $y^{\prime}$ and $z^{\prime}$ correspond to a pixel size. The value of $\theta\left(y^{\prime}, z^{\prime}\right)$ (used in the Bragg law to get the wavelength, as in step 2) is computed for each line out (in the spectral direction) and stored in memory to re-index each pixel of the image with respect to the wavelength scale using the relation

$$
\sin \theta\left(y^{\prime}, z^{\prime}\right)=\left(\sin ^{2} \theta_{0}+\left(y^{\prime} \cos \theta_{0} / R_{h}\right)\right) / \sqrt{\sin ^{2} \theta_{0}+\left(y^{\prime} / R_{h}\right)^{2}+\left(z^{\prime} / 2 R_{h}\right)^{2}}
$$

4. Reconstructed picture is saved while line outs can be extracted directly from the software.

The effective angle $\psi$ between a radiation incident on the crystal and the target plane is also taken into account and corrected with respect to the spatial resolution coordinate using $z=\frac{z^{\prime}}{\cos \psi}$, where $\psi$ is the angle between expanding plasma direction and target-to-crystal direction $\left(\psi=25^{\circ}\right.$ during the present experiment).

\section{RESULTS}

The HIDEX prototype has been tested at PALS kilo-joule laser facility, Prague. A bulk aluminium target was heated with a $23 \mathrm{~J}$ pulse of 300 ps duration at frequency-tripled laser light $(0.44 \mu \mathrm{m})$ and a focal spot size of $80 \mu \mathrm{m}$ resulting in an intensity $I=1.5 \times 10^{15} \mathrm{~W} / \mathrm{cm}^{2}$ on the target. The desired spectral range (set to record $\mathrm{Ly}_{\alpha}$ and $J$ satellites of Al plasma, between 7.1 and $7.3 \AA$ ) and the crystal radius of curvature $\left(\mathrm{R}_{h}=76.6 \mathrm{~cm}\right)$ constrain the target-to-crystal-to-detector distance, which was $\mathrm{L}_{0}=131.3 \mathrm{~mm}$ in our experiment. The cylindrically bent crystal of quartz (10-10) used had an active area of $4 \times 1 \mathrm{~cm}^{2}$. The spectra were recorded using Kodak HS800 X-ray films. Figure 3 shows a result of the procedure described in Sect. 3. Each curve is a line out of the reconstructed image at different positions from the target surface. During the test, the prototype did not benefit from the goniometers behind the crystal (the tilt broadened the lines and produced a small asymmetry) as well as from a proper shielding (it introduced a high background that deteriorates the contrast). Despite its prototype state, two remarkable features can be seen. First, $\mathrm{Ly}_{\alpha}$ and $\mathrm{J}$ satellites [7] lines are clearly observed. Second, 

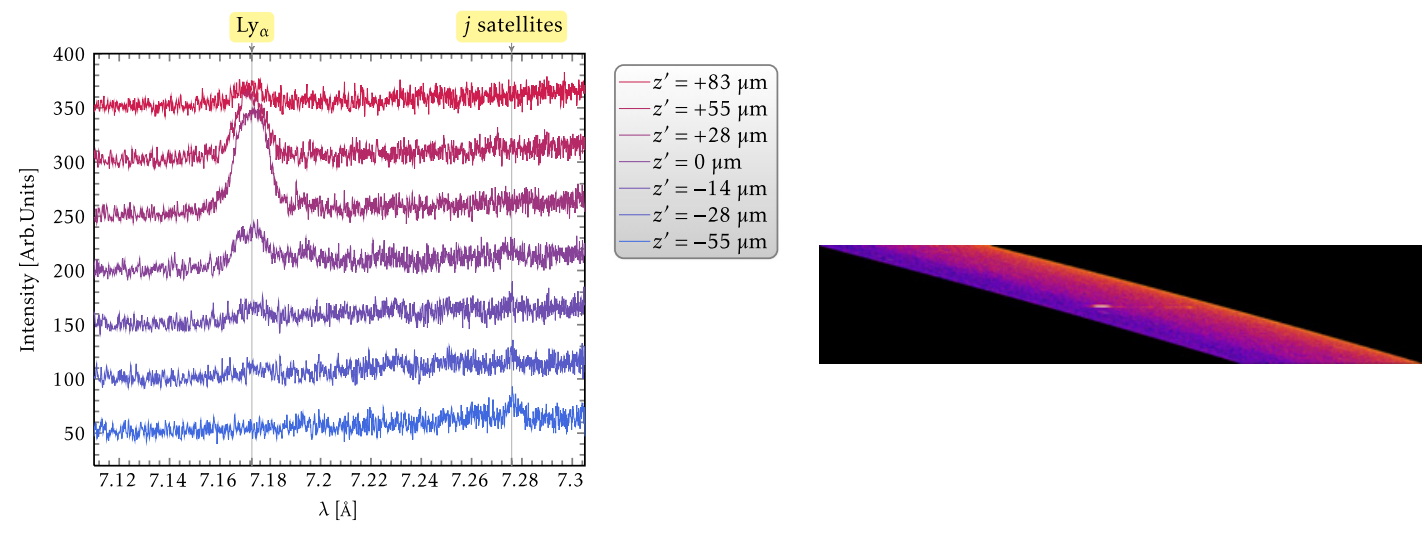

Figure 3. Left: a set of experimental results from the HIDEX prototype. $z^{\prime}$ is the distance of the line out with respect to the surface of the target. Each curve has been shifted for clarity. Right: a reconstructed spectrum in synthetic colors.

emission from inside the crater is resolved (negative distance on Figure 2) and shows a disappearance of resonance lines while the satellites are more intense. This behaviour has already been observed in similar conditions [8] indicating the observation of the dense and cold aluminium plasma inside the crater.

\section{CONCLUSION}

We describe the prototype of the HIDEX spectrometer based on the vertical Johann configuration of a cylindrically bent crystal. It uses a motorized rotation stage whose axis is coincident with the crystal surface in order to speed up the alignment procedure while retaining a high accuracy. A CCD can be used as detector in such a manner that the spectrometer complies with requirements of high repetition rate laser facilities. The test of the prototype at PALS shows $\mathrm{Ly}_{\alpha}$ and $\mathrm{j}$ satellites emission from laser-irradiated bulk aluminium target. Disappearance of resonance line in the crater indicates that emission from the dense and cold aluminium plasma inside the crater, about $50 \mu \mathrm{m}$ below the surface, has been observed. These results validate the entirely new alignment procedure of this instrument.

This research has been supported in part by the Czech Science Foundation Grant P205/10/0814 and the Czech Ministry of Education projects No. LM2010014 (PALS RI).

\section{References}

[1] J. D. Lindl et al., Physics of Plasmas, 11, 339-491 (2004)

[2] W. B. Hubbard, Science 214, 145-149 (1981)

[3] B. Barbrel et al., Phys. Rev. Lett. 102, 165004 (2009)

[4] F. B. Rosmej, Highly Charged Ions, 267-343 (editors R. Hutton et al., Taylor and Francis, 2011)

[5] E. Galtier et al., Phys. Rev. Lett. 106, 164801 (2011)

[6] O. Renner et al., Rev. Sci. Instr. 68, 2393-2403 (1997)

[7] Yu. Ralchenko et al., NIST Atomic Spectra Database (version 3.1.5), [Online]. Available: http://physics.nist.gov/asd3 (2010, August 22)

[8] O. Renner et al., Appl. Phys. Lett. 79, 177-179 (2001) 